\section{CT features of thyroid nodules with isolated macrocalcifications detected by ultrasonography}

\author{
Wooyul Paik ${ }^{1}$, Dong Gyu Na ${ }^{1,2}$, Hye Yun Gwon ${ }^{1}$, Jinna Kim ${ }^{3}$ \\ 'Department of Radiology, Gangneung Asan Hospital, University of Ulsan College of \\ Medicine, Gangneung; '²Department of Radiology, Human Medical Imaging and Intervention \\ Center, Seoul; ${ }^{3}$ Department of Radiology, Yonsei University College of Medicine, Seoul, Korea
}

Purpose: A thyroid nodule with an isolated macrocalcification is visualized as a calcified nodule with complete posterior shadowing on ultrasonography (US). This study aimed to determine the computed tomography (CT) features of isolated macrocalcifications detected using US.

Methods: This study included 20 patients who had thyroid nodules with isolated macroalcifications and underwent neck CT or chest CT. The patients were enrolled from a sample of 82 patients with isolated macrocalcifications detected by US drawn from 7,142 consecutive patients who underwent thyroid biopsy at two institutions. We evaluated the CT features of nodules with isolated macrocalcifications and categorized them as central or rim calcifications. We assessed the nodule size and the frequency of nondiagnostic fine-needle aspiration (FNA) results and malignant tumors according to the CT features of isolated macrocalcifications.

Results: CT scans showed central calcifications in 18 (90.0\%) and rim calcifications in two $(10.0 \%)$ of the 20 nodules with isolated macrocalcifications. Among the 18 nodules with central isolated macrocalcifications, complete compact calcification was found in six nodules and partial coarse calcification in 12 nodules. In 18 nodules with central isolated macrocalcifications, the nondiagnostic FNA rate and frequency of malignant tumors were not significantly different between complete and partial central calcifications ( $P=0.620$ and $P=0.999$, respectively). Malignant tumors were only found in nodules with central isolated macrocalcifications.

Conclusion: The majority of nodules with isolated macrocalcifications showed central calcifications on CT. Thyroid nodules with isolated macrocalcifications detected by US should not be classified as having a type of rim or peripheral calcification.

Keywords: Thyroid nodule; Calcinosis; Diagnosis; Ultrasonography;

Multidetector computed tomography

\section{Introduction}

Ultrasonography (US) plays an essential role in estimating the malignancy risk of a thyroid nodule and in the choice of a subsequent management strategy [1]. Although macrocalcifications may be

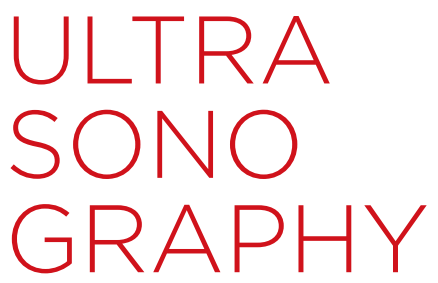

ORIGINAL ARTICLE

https://doi.org/10.14366/usg.19045 pISSN: 2288-5919 • eISSN: 2288-5943 Ultrasonography 2020;39:130-136

Received: August 15, 2019 Revised: September 26, 2019 Accepted: October 23, 2019

Correspondence to: Dong Gyu Na, MD, PhD, Department of Radiology, Gangneung Asan Hospital, University of Ulsan College of Medicine, 38 Bangdong-gil, Gangneung 25440, Korea

Tel. +82-33-610-4310

Fax. +82-33-610-3490 E-mail: nndgna@gmail.com

This is an Open Access article distributed under the terms of the Creative Commons Attribution NonCommercial License (http://creativecommons.org/ licenses/by-nc/4.0/) which permits unrestricted noncommercial use, distribution, and reproduction in any medium, provided the original work is properly cited.

Copyright $(2020$ Korean Society of Ultrasound in Medicine (KSUM)

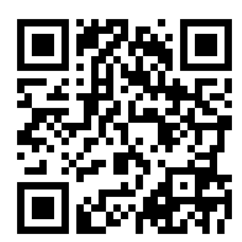

How to cite this article:

Paik W, Na DG, Gwon HY, Kim J. CT features of thyroid nodules with isolated macrocalcifications detected by ultrasonography. Ultrasonography. 2020 Apr;39(2):130-136. 
associated with an increased risk of malignancy, the malignancy rate in nodules with macrocalcifications has been found to be highly variable, meaning that macrocalcifications are not a highly specific predictor of malignancy [2-5]. An isolated macrocalcification is defined as a calcified nodule with complete posterior acoustic shadowing in which no soft tissue component can be identified due to dense shadowing on US [6]. A US lexicon for isolated macrocalcifications has not been established, and the presence of isolated macrocalcifications has only been considered as part of the risk stratification of nodules in the Korean Thyroid Imaging Reporting and Data System (K-TIRADS) [7,8] and in the American College of Radiology (ACR) Thyroid Imaging Reporting and Data System (TI-RADS) [8-10]. Previous studies [11-14] classified isolated macrocalcifications as a type of rim or peripheral calcification. However, the details of an isolated macrocalcification cannot be evaluated using US alone due to the dense posterior shadowing, which causes challenges in incorporating nodules with isolated macrocalcifications into the risk stratification of thyroid nodules [710].

Meanwhile, computed tomography (CT) has the advantage of enabling detailed evaluations of isolated macrocalcifications. A previous study [6] reported that isolated macrocalcifications may present as entirely calcified nodules on CT; however, there has been little research into the $\mathrm{CT}$ features of isolated macrocalcifications. Therefore, this study aimed to determine the CT features of thyroid nodules with isolated macrocalcifications detected by US.

\section{Materials and Methods}

The institutional review board approved this retrospective study, and the requirement to obtain informed consent was waived.

\section{Patients}

This study included 20 patients ( 17 women, 3 men; mean age, $61.1 \pm 11.6$ years) who had thyroid nodules with isolated macrocalcifications and underwent neck CT or chest CT (Fig. 1). Thyroid nodules with isolated macrocalcifications were defined as isolated calcified nodules with complete posterior acoustic shadowing in which no solid component was obviously identified within the nodules on US (Figs. 2-4). The enrolled patients were selected from 82 patients with isolated macrocalcifications detected by US at two institutions. Forty-four patients with isolated macrocalcifications were found in the cohort data (dataset 1 , $n=4,081)$ of patients with thyroid nodules at one institution; details about this cohort have previously been published [6]. Thirtyeight patients with isolated macrocalcifications were found in the cohort data (dataset $2, n=3,061$ ) of patients with thyroid nodules in whom fine-needle aspiration (FNA) or core needle biopsy (CNB) was performed from January 2011 to June 2018 at another institution. In dataset 1 , nodules with isolated macrocalcifications

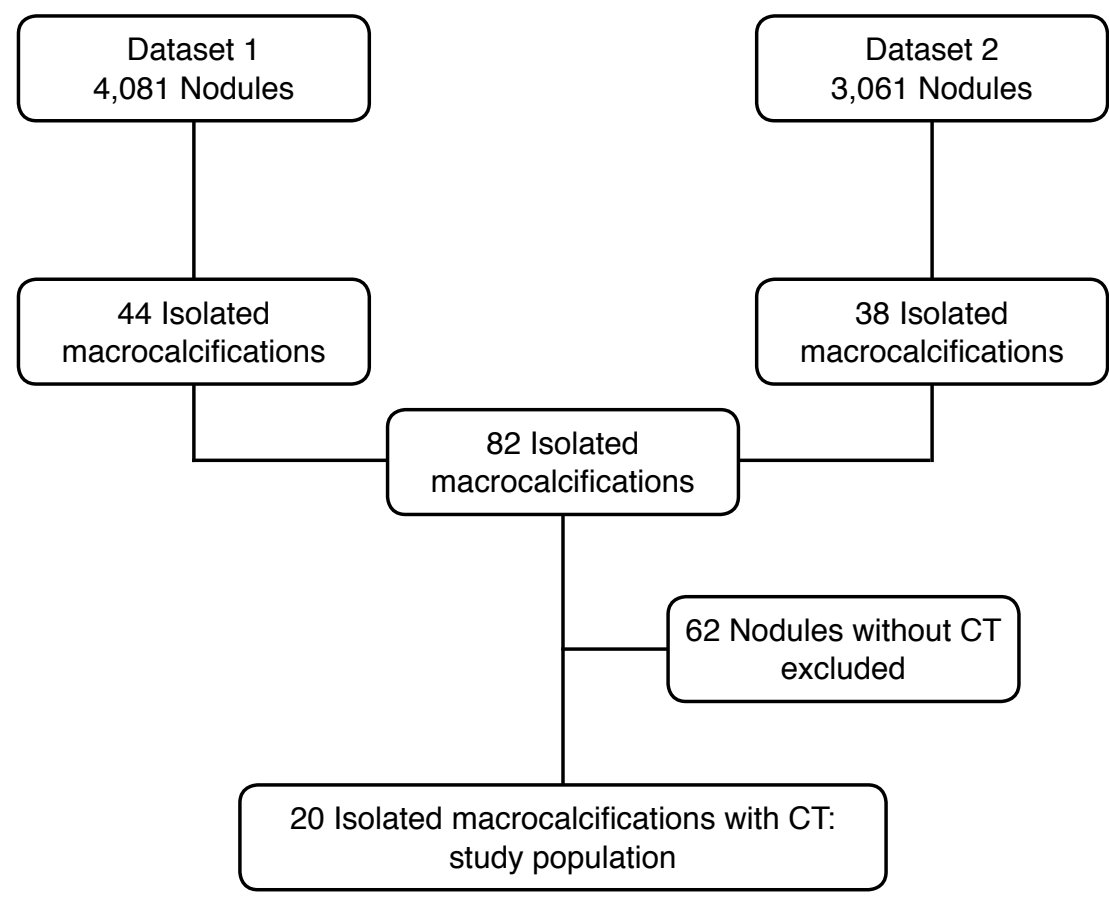

Fig. 1. Flow diagram of patient enrollment. $\mathrm{CT}$, computed tomography. 
were retrospectively determined by reviewing the thyroid US of 408 patients for which the word "macrocalcification" was used in the radiology report of thyroid US [6]. In dataset 2, nodules with isolated macrocalcifications were retrospectively determined by reviewing the US of all patients. Among the 82 patients with isolated macrocalcifications detected by US at the two institutions, 62 patients who did not undergo neck or chest CT were excluded from this study (Fig. 1).

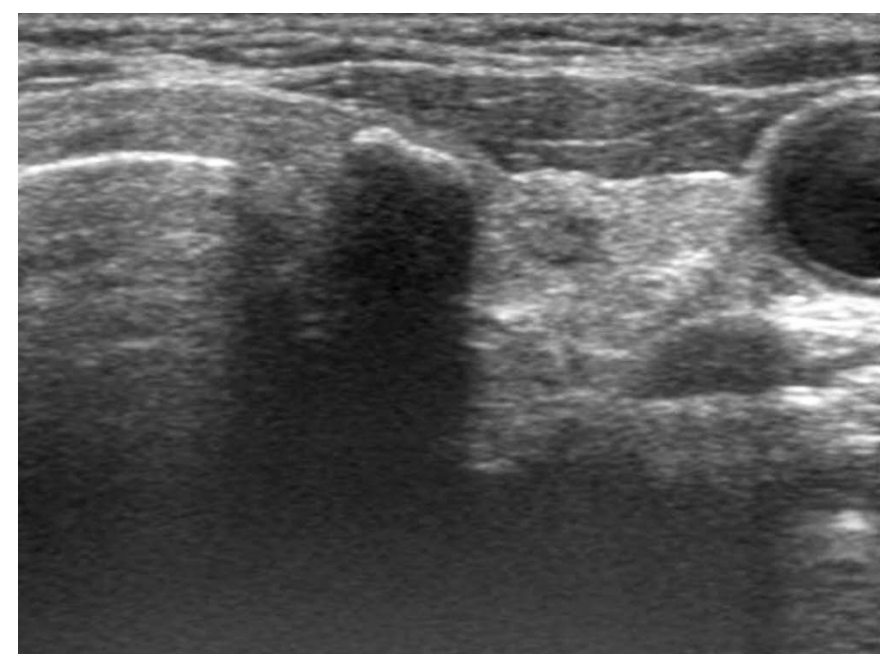

A

Fig. 2. Thyroid nodule with an isolated macrocalcification in a 70-year-old woman.

A. Transverse ultrasonography (US) shows a calcified nodule $(6 \mathrm{~mm})$ in the left lobe, and the posterior margin of the calcified nodule is not visualized due to strong posterior acoustic shadowing. B. Axial unenhanced computed tomography shows a complete compact central calcification in a case where an isolated macrocalcification was detected by US.

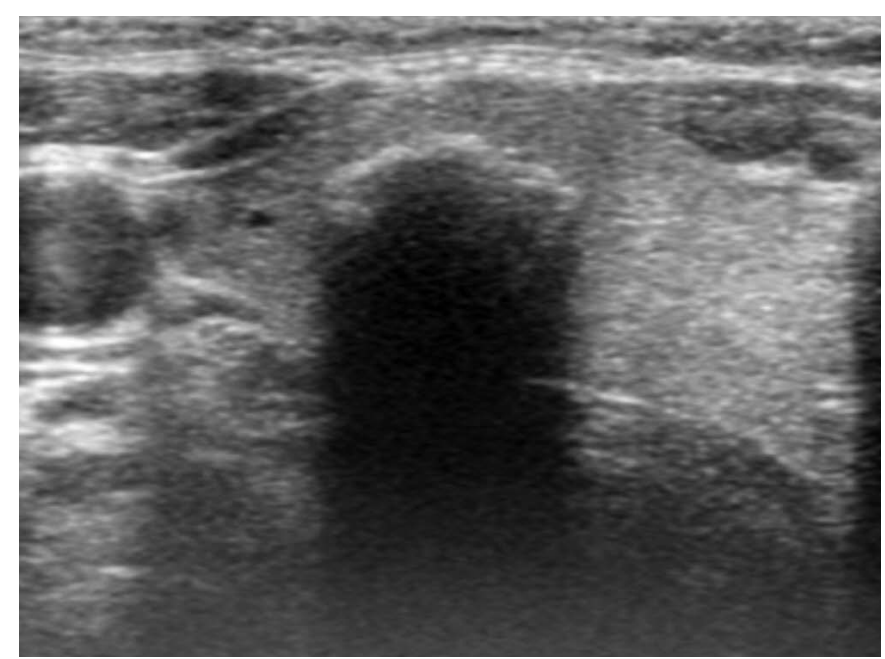

A

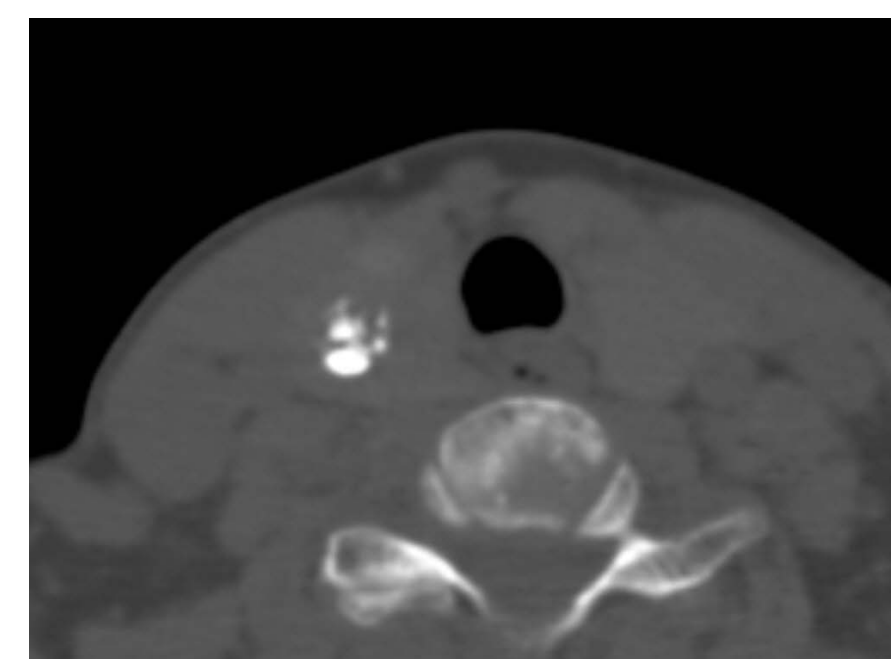

B

Fig. 3. Thyroid nodule with an isolated macrocalcification in a 59-year-old woman.

A. Transverse ultrasonography (US) shows a calcified nodule $(12 \mathrm{~mm})$ with strong posterior acoustic shadowing in the right lobe. B. Axial unenhanced computed tomography shows a partial coarse central calcification in a case where an isolated macrocalcification was detected by US. 


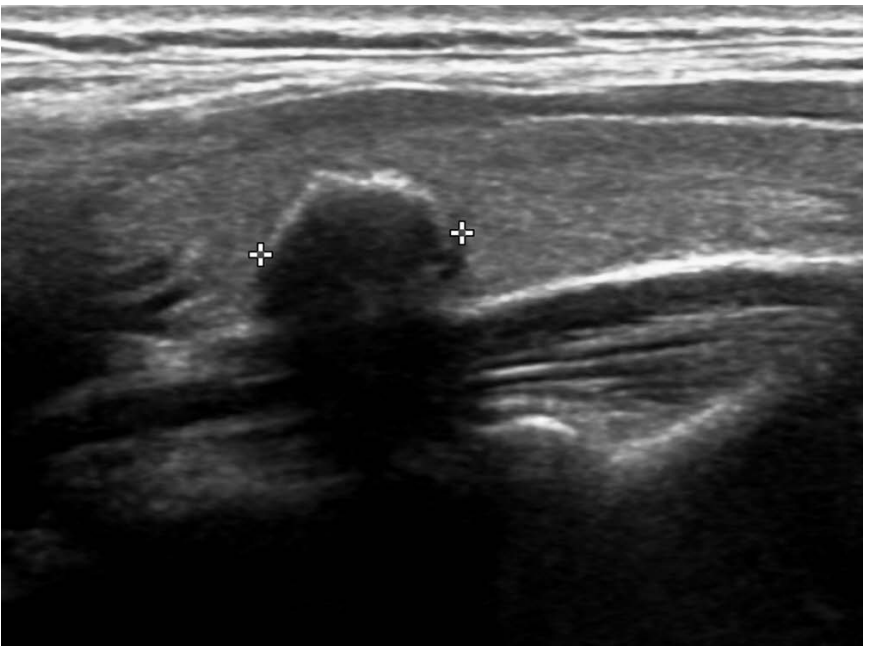

A

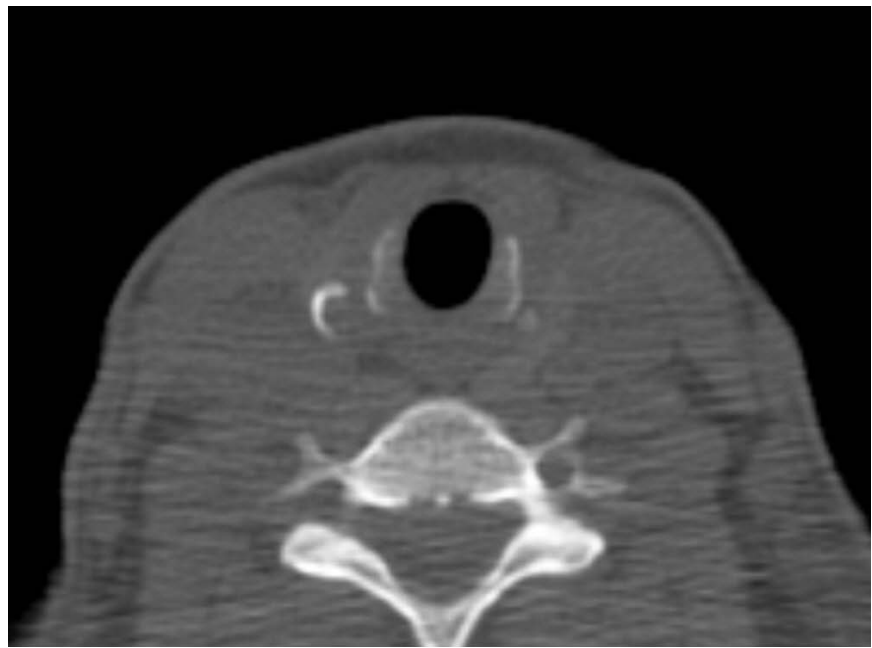

B

Fig. 4. Thyroid nodule with an isolated macrocalcification in a 50-year-old woman.

A. Longitudinal ultrasonography (US) shows a calcified nodule $(10 \mathrm{~mm})$ with strong posterior acoustic shadowing in the right lobe. B. Axial unenhanced computed tomography shows a curvilinear incomplete rim calcification at the right margin of an isolated macrocalcification that was detected by US.

were determined by surgery or based on benign findings on FNA or CNB.

\section{US and CT Examinations}

High-resolution US examinations were performed using a 10- to 12MHz linear-array transducer (AplioXG, Toshiba, Otawarashi, Japan) and 5- to 12-MHz linear-array transducers (iU22 or EPIQ7, Philips Medical Systems, Bothell, WA, USA). Neck CT scans were obtained in 11 patients and chest $\mathrm{CT}$ scans in nine patients. For $\mathrm{CT}$ examinations, 64-channel multidetector CT scanners were used. Unenhanced neck CT was performed to evaluate the calcified mass before biopsy in seven patients, and both unenhanced and contrast-enhanced neck CT was performed as part of the preoperative evaluation in four patients diagnosed with thyroid cancer. Unenhanced chest CT was obtained in four patients and contrast-enhanced chest CT in five patients for the evaluation of lung lesions. The neck CT scans were reconstructed into axial images with scan parameters of 2- to 4-mm slice thickness, $512 \times 512$ matrix, and a 22- to 25 $\mathrm{cm}$ field of view (FOV). The chest CT scans were reconstructed into axial images with scan parameters of a $2.5-$ to $5-\mathrm{mm}$ slice thickness, a $512 \times 512$ matrix, and a $33-$ to $43-\mathrm{cm}$ FOV. The unenhanced neck and chest CT scans were reviewed to evaluate isolated macrocalcifications in 15 patients, and enhanced chest CT scans were reviewed in five patients.

\section{US-Guided FNA and CNB Procedures}

US-guided FNA was performed in the conventional manner using a 21- to 23-gauge needle and at least two samples were obtained from each nodule [15]. Direct smears or liquid-based cytology was used for the preparation of FNA specimens. The specimen was smeared on a slide and immediately fixed in $95 \%$ ethanol using the direct smear method. In the liquid-based cytology method, the specimens were prepared using the ThinPrep 2000 processor (Hologic Co., Marlborough, MA, USA).

US-guided CNB was performed using a free-hand technique and a disposable 18-gauge, double-action spring-activated needle (1.1-cm excursion, TSK Acecut, Create Medic, Yokohama, Japan) as described previously $[6,16]$. Each specimen was immediately fixed in $10 \%$ neutral buffered formalin solution and stained in the standard manner for a histopathologic examination. The interpretation of FNA or CNB was based on the Bethesda system for reporting thyroid cytopathology [17] and the six categories of a CNB pathology reporting system [18]. After the patients underwent biopsy, we immediately compressed the biopsy site, and the patients were observed as they performed manual self-compression of the biopsy site for 20-30 minutes.

\section{Image Analysis and Statistics}

US were retrospectively reviewed by an experienced radiologist (D.G.N.) with 21 years of experience in performing thyroid US and interventional procedures. A reviewer who had no previous knowledge of the FNA results or final diagnoses determined the presence of isolated macrocalcifications from the database of patients' US. The CT features of the included nodules with 
isolated macrocalcifications were retrospectively evaluated by two radiologists (D.G.N. and W.P.) through consensus in a blind fashion. The $\mathrm{CT}$ features of the isolated macrocalcifications were categorized into central and rim calcifications. A central calcification was defined as a calcified nodule with an intranodular macrocalcification located in its center. Central calcifications were further subcategorized as either complete calcifications (compact entire calcifications) or partial calcifications (coarse partial calcification with an obvious non-calcified soft tissue component within the calcified nodule). A rim (peripheral) calcification was defined as a calcified nodule with complete or incomplete curvilinear rim calcification. The $\mathrm{CT}$ features of central isolated macrocalcifications were evaluated on axial CT scans with a wide window width (1,500 Hounsfield units) to assess the internal architecture of the calcified nodules.

We assessed the nodule size and frequency of nondiagnostic FNA results and malignant tumors according to the $\mathrm{CT}$ features of isolated macrocalcifications. The Mann-Whitney $U$ test was used to compare the mean nodule size and the Fisher exact test was used to compare the nondiagnostic FNA rate between complete and partial central calcifications. Statistical analyses were performed using SPSS for Windows version 24.0 (IBM Corp., Armonk, NY, USA). A significant difference was defined as a P-value of $<0.05$.

\section{Results}

\section{Demographic Data}

CT scans were obtained in 20 of the 82 patients with isolated macrocalcifications (24.4\%). The size of the 20 nodules ranged from 6 to $20 \mathrm{~mm}$ (mean size, $12.3 \pm 3.4 \mathrm{~mm}$ ). Among the 20 nodules with isolated macrocalcifications, both FNA and CNB were performed simultaneously in nine (45.0\%), FNA alone in $10(50.0 \%)$, and CNB alone in one $(5.0 \%)$ in the initial biopsy procedure. The initial FNA results of the 19 nodules were nondiagnostic $(n=9,47.4 \%)$, benign $(n=6,31.6 \%)$, atypia of undetermined significance/follicular lesion of undetermined significance $(n=2,10.5 \%)$, suspicious for malignancy $(n=1,5.3 \%)$, and malignant $(n=1,5.3 \%)$. The initial CNB results of the 10 nodules were nondiagnostic $(n=1,10.0 \%)$, benign $(n=4$, $40.0 \%)$, indeterminate $(n=4,40.0 \%)$, and malignant $(n=1,10 \%)$. Final diagnoses were obtained in 12 of the 20 nodules $(60.0 \%)$, and eight nodules showed inconclusive FNA or CNB results. Malignant tumors were found in four of the 20 nodules $(20.0 \%)$, of which three were conventional papillary thyroid carcinomas (PTC) and one was found to be invasive encapsulated follicular variant PTC.

\section{CT Features of Isolated Macrocalcifications}

Central calcifications were found on the CT of 19 of the 20 nodules $(90.0 \%)$ with isolated macrocalcifications, and rim calcifications were found in two (10.0\%) (Table 1). Among the 18 nodules with central calcifications, complete calcifications were found in six nodules (Fig. 1) and partial calcifications in 12 nodules (Fig. 2). The two nodules with rim calcifications showed incomplete rim calcifications, and no complete rim calcifications were observed (Fig. 3).

\section{Nodule Size, Nondiagnostic FNA Results, and Frequency of Malignant Tumors according to Isolated Macrocalcification Type}

In the 18 nodules with central isolated macrocalcifications, the mean size of nodules belonging to the partial calcification subtype was larger than that of nodules belonging to the complete calcification subtype, but this difference was statistically insignificant $(\mathrm{P}=0.098)$ (Table 2). Nondiagnostic FNA results were found in nine of the 19 nodules that underwent FNA (47.4\%). The nondiagnostic rate was similar in central calcifications (47.1\%) and rim calcifications (50\%), and there was no significant difference in the nondiagnostic rate between complete and partial central calcifications ( $P=0.620$ ) (Table 2). Malignant tumors were only found in four of the 18 nodules with a central isolated macrocalcification (22.2\%). There was no significant difference in the frequency of malignant tumors between complete and partial central calcifications ( $P=0.999)$.

Table 1. CT features of isolated macrocalcifications $(n=20)$

\begin{tabular}{lc}
\hline \multicolumn{1}{c}{ Type of calcification } & No. of nodules (\%) \\
\hline Central & $18(90.0)$ \\
Complete & 6 \\
Partial & 12 \\
Rim & $2(10.0)$ \\
\hline
\end{tabular}

CT, computed tomography.

Table 2. Nodule size, nondiagnostic FNA results, and frequency of malignant tumors according to CT types of isolated macrocalcifications

\begin{tabular}{lccc}
\hline Type of calcification & $\begin{array}{c}\text { Nodule size } \\
(\mathrm{mm})\end{array}$ & $\begin{array}{c}\text { Nondiagnostic } \\
\text { FNA result }\end{array}$ & $\begin{array}{c}\text { No. of malignant } \\
\text { tumors }\end{array}$ \\
\hline All $(\mathrm{n}=20)$ & $12.3 \pm 3.4$ & $9(47.4)^{\mathrm{a})}$ & $4(20.0)$ \\
Central $(\mathrm{n}=18)$ & $12.3 \pm 3.5$ & $8(47.1)^{\mathrm{a})}$ & $4(22.2)$ \\
Complete $(\mathrm{n}=6)$ & $10.5 \pm 3.4$ & $2(33.3)$ & $1(16.7)$ \\
Partial $(\mathrm{n}=12)$ & $13.3 \pm 3.4$ & $6(54.4)^{\mathrm{a})}$ & $3(25.0)$ \\
$\operatorname{Rim}(\mathrm{n}=2)$ & $11.5 \pm 2.1$ & $1(50.0)$ & 0 \\
\hline
\end{tabular}

Values are presented as mean \pm standard deviation or number (\%).

FNA, fine-needle aspiration; CT, computed tomography.

${ }^{\text {a) }}$ FNA was not performed in 1 nodule. 


\section{Discussion}

Our study demonstrated that the majority of nodules with isolated macrocalcifications (90.0\%) showed central calcifications on $C T$, which suggests that thyroid nodules with isolated macrocalcifications should not be classified as having a type of rim or peripheral calcification. Our study also showed that the three distinct types of macrocalcifications (central complete, central partial, and rim) may be similar in terms of showing the US feature of isolated macrocalcifications.

In the US lexicon, macrocalcifications are defined as echogenic foci larger than $1 \mathrm{~mm}$ with posterior acoustic shadowing, and this definition is consistent across the Korean Society of Thyroid Radiology (KSThR), European Thyroid Association (ETA), and ACR guidelines $[7,9,19]$. However, isolated macrocalcifications have not been clearly defined in the published guidelines, and were categorized as a type of rim or peripheral calcification in previous studies [11-14]. Based on the results of our study, an isolated macrocalcification should be differentiated from a rim calcification, which is defined as a peripheral curvilinear echogenic rim with or without posterior shadowing. Rim calcifications with posterior shadowing should be defined as cases where any part of the internal content can be visualized despite accompanying dense posterior shadowing.

Distinguishing isolated macrocalcifications from rim calcifications may be useful in the diagnosis and management of thyroid nodules. First, clarifying the definition of rim calcifications may improve interobserver agreement in the interpretation of calcified nodules, because interobserver variability is closely related to the clarity of the US lexicon. Second, the malignancy risk and diagnostic value of rim calcification might be further elucidated. Previous studies $[2,3,5,20]$ showed conflicting results regarding the malignancy risk of lesions with rim calcifications and the association of rim calcifications with malignancy, and these discrepancies might have been affected by differences in the definition of rim calcifications. Third, more cautious FNA or application of CNB is necessary for nodules with isolated macrocalcifications because the nondiagnostic FNA rate is very high [6].

The US lexicon plays an important role in the risk stratification of thyroid nodules for malignancy. Isolated macrocalcifications are categorized as intermediate suspicious nodules in the K-TIRADS $[7,8]$ and as moderately suspicious nodules in the ACR TI-RADS [810]. However, it is not specified in the risk stratification of thyroid nodules in other thyroid society guidelines. A previous study [6] reported that the risk of malignancy ranged from $11.4 \%$ to $16.1 \%$ in all nodules with isolated macrocalcifications, and was $16.7 \%$ in nodules $(\geq 1 \mathrm{~cm})$ with final diagnoses. A recent study [14] reported that the risk of malignancy of nodules with completely shadowed peripheral calcifications was $38.5 \%$. In our study, the CT pattern of central isolated macrocalcifications showed a similar frequency of malignant tumors. However, our study had limited ability to assess the malignancy risk of each $\mathrm{CT}$ calcification pattern due to the inclusion of a small number of patients. In our study, the nondiagnostic FNA rate was similar between complete and partial central isolated macrocalcifications. However, it remains possible that a completely calcified nodule might increase the risk of a nondiagnostic FNA result because needle penetration may be more difficult in a totally calcified nodule than in a partially calcified nodule.

There are several limitations to this study. First, the number of included patients was small, and selection bias was present because CT scans were not obtained for many of the patients with isolated macrocalcifications. Second, the statistical results are limited because of the small sample size of our study. Third, the retrospective assessment of US by one interpreter is an inherent limitation regarding the interpretation of isolated macrocalcifications. Third, further investigation is necessary to determine whether the nondiagnostic FNA rate and risk of malignancy may differ according to the $\mathrm{CT}$ features of isolated macrocalcifications.

In conclusion, the majority of thyroid nodules with isolated macrocalcifications $(90.0 \%)$ showed central calcifications on CT. Therefore, thyroid nodules with isolated macrocalcifications detected by US should not be classified as having a type of rim or peripheral calcification.

ORCID: Wooyul Paik: https://orcid.org/0000-0001-9617-6227; Dong Gyu Na: https://orcid.org/0000-0001-6422-1652; Hye Yun Gwon: https://orcid.org/00000002-6899-0309; Jinna Kim: https://orcid.org/0000-0002-9978-4356

\section{Author Contributions}

Conceptualization: Na DG. Data acquisition: Gwon HY, Kim J, Na DG. Data analysis or interpretation: Paik W, Na DG. Drafting of the manuscript: Paik W, Na DG. Critical revision of the manuscript: Paik W, $\mathrm{Na}$ DG. Approval of the final version of the manuscript: all authors.

\section{Conflict of Interest}

No potential conflict of interest relevant to this article was reported.

\section{Acknowledgments}

This research was supported by the Medical Research Promotion Program through the GangNeung Asan Hospital funded by the Asan Foundation (2018-C03). 


\section{References}

1. Ha EJ, Lim HK, Yoon JH, Baek JH, Do KH, Choi M, et al. Primary imaging test and appropriate biopsy methods for thyroid nodules: guidelines by Korean Society of Radiology and national evidence-based healthcare collaborating agency. Korean J Radiol 2018;19:623-631.

2. Frates $M C$, Benson $C B$, Doubilet $P M$, Kunreuther $E$, Contreras $M$, Cibas ES, et al. Prevalence and distribution of carcinoma in patients with solitary and multiple thyroid nodules on sonography. J Clin Endocrinol Metab 2006;91:3411-3417.

3. Moon WJ, Jung SL, Lee JH, Na DG, Baek JH, Lee YH, et al. Benign and malignant thyroid nodules: US differentiation-multicenter retrospective study. Radiology 2008;247:762-770.

4. Lu Z, Mu Y, Zhu H, Luo Y, Kong Q, Dou J, et al. Clinical value of using ultrasound to assess calcification patterns in thyroid nodules. World J Surg 2011;35:122-127.

5. Na DG, Baek JH, Sung JY, Kim JH, Kim JK, Choi YJ, et al. Thyroid Imaging Reporting and Data System risk stratification of thyroid nodules: categorization based on solidity and echogenicity. Thyroid 2016;26:562-572.

6. Na DG, Kim DS, Kim SJ, Ryoo JW, Jung SL. Thyroid nodules with isolated macrocalcification: malignancy risk and diagnostic efficacy of fine-needle aspiration and core needle biopsy. Ultrasonography 2016;35:212-219.

7. Shin JH, Baek JH, Chung J, Ha EJ, Kim JH, Lee YH, et al. Ultrasonography diagnosis and imaging-based management of thyroid nodules: revised Korean Society of Thyroid Radiology consensus statement and recommendations. Korean J Radiol 2016;17:370-395.

8. Yoon SJ, Na DG, Gwon HY, Paik W, Kim WJ, Song JS, et al. Similarities and differences between Thyroid Imaging Reporting and Data Systems. AJR Am J Roentgenol 2019;213:W76-W84.

9. Tessler FN, Middleton WD, Grant EG, Hoang JK, Berland LL, Teefey SA, et al. ACR Thyroid Imaging, Reporting and Data System (TIRADS): white paper of the ACR TI-RADS Committee. J Am Coll Radiol 2017;14:587-595.

10. Middleton WD, Teefey SA, Reading CC, Langer JE, Beland MD, Szabunio MM, et al. Comparison of performance characteristics of American College of Radiology TI-RADS, Korean Society of Thyroid
Radiology TIRADS, and American Thyroid Association guidelines. AJR Am J Roentgenol 2018;210:1148-1154.

11. Yoon DY, Lee JW, Chang SK, Choi CS, Yun EJ, Seo YL, et al. Peripheral calcification in thyroid nodules: ultrasonographic features and prediction of malignancy. J Ultrasound Med 2007;26:13491355.

12. Kim BM, Kim MJ, Kim EK, Kwak JY, Hong SW, Son EJ, et al. Sonographic differentiation of thyroid nodules with eggshell calcifications. J Ultrasound Med 2008;27:1425-1430.

13. Park YJ, Kim JA, Son EJ, Youk JH, Kim EK, Kwak JY, et al. Thyroid nodules with macrocalcification: sonographic findings predictive of malignancy. Yonsei Med J 2014;55:339-344.

14. Malhi HS, Velez E, Kazmierski B, Gulati M, Deurdulian C, Cen SY, et al. Peripheral thyroid nodule calcifications on sonography: evaluation of malignant potential. AJR Am J Roentgenol 2019;213:672-675.

15. Lee YH, Baek JH, Jung SL, Kwak JY, Kim JH, Shin JH, et al. Ultrasoundguided fine needle aspiration of thyroid nodules: a consensus statement by the Korean Society of Thyroid Radiology. Korean J Radiol 2015;16:391-401.

16. Na DG, Baek JH, Jung SL, Kim JH, Sung JY, Kim KS, et al. Core needle biopsy of the thyroid: 2016 consensus statement and recommendations from Korean Society of Thyroid Radiology. Korean J Radiol 2017;18:217-237.

17. Cibas ES, Ali SZ. The Bethesda System for Reporting Thyroid Cytopathology. Thyroid 2009;19:1159-1165.

18. Jung CK, Min HS, Park HJ, Song DE, Kim JH, Park SY, et al. Pathology reporting of thyroid core needle biopsy: a proposal of the Korean Endocrine Pathology Thyroid Core Needle Biopsy Study Group. J Pathol Transl Med 2015;49:288-299.

19. Russ G, Bonnema SJ, Erdogan MF, Durante C, Ngu R, Leenhardt L. European Thyroid Association guidelines for ultrasound malignancy risk stratification of thyroid nodules in adults: the EU-TIRADS. Eur Thyroid J 2017;6:225-237.

20. Middleton WD, Teefey SA, Reading CC, Langer JE, Beland MD, Szabunio MM, et al. Multiinstitutional analysis of thyroid nodule risk stratification using the American College of Radiology Thyroid Imaging Reporting and Data System. AJR Am J Roentgenol 2017;208:1331-1341. 